\title{
ON THE CONSISTENCY THEOREM IN MATRIX SUMMABILITY
}

\author{
ROBERT E. ATALLA
}

\begin{abstract}
We give a generalization of the consistency theorem for bounded convergence fields. The space $c$ of convergent sequences is replaced by a space of more general type. Applications of the generalized consistency theorem are made to multiplicative summability theory. In particular we give conditions under which a generalized convergence field is an algebra.
\end{abstract}

1. Introduction. In 1933, Mazur and Orlicz [M-O $\mathbf{O}_{1}$ ] announced their famous result that if the bounded convergence field of a regular matrix $A$ is contained in that of another regular matrix $B$, then the matrices are consistent on the first field, i.e., $A-\lim x=B$ - $\lim x$ whenever $x$ is in the bounded convergence field of $A$. (Their proof, based on functional analysis, was published in $\left[\mathbf{M}-\mathbf{O}_{2}\right]$.) Later Brudno [B] gave a complicated but elementary proof. Independently of each other, G. M. Petersen $\left[\mathbf{P}_{\mathbf{1}}\right]$ and Erdös-Piranian [E-P] were able to isolate a basic principle used implicitly by Brudno, and to use this principle to give easy proofs of the consistency theorem.

In $\S 2$ we give a formulation of the basic principle which, combined with certain facts concerning the topology of the space $\beta N \backslash N$ (to be explained in $\S 2$ ), yields in $\S 3$ a generalization of the consistency theorem. In this generalization the space $c$ of convergent sequences is replaced by a space of more general type, subject to certain conditions. Most importantly the space must, in a sense to be specified, be 'small' as a subspace of the space of bounded sequences. As an application of the generalized consistency theorem we derive in $\S 4$ generalizations of known results on the multiplicative behavior of regular matrices.

Basic to our method is the device of representing a regular matrix as a linear operator on $C(\beta N \backslash N)$, as explained below. (See [A-B], [A].)

2. The basic lemma. Our version of the basic principle mentioned in the introduction is given in Lemma 2.4. We have stated it in a form

Received by the editors December 12, 1971.

AMS 1970 subject classifications. Primary 40C05, 40D20, 40D25.

Key words and phrases. Convergence field, inclusion theorem, consistent matrix methods, multipicative matrix method, $\beta N$.

(c) American Mathematical Society 1972 
convenient for our own use, but the details of the proof are gleaned from $\left[\mathbf{P}_{1}\right],\left[\mathbf{P}_{2}\right]$ and $[\mathbf{E}-\mathbf{P}]$. For completeness we give a detailed proof.

2.1. Notation. $C^{*}(N)$ is the space of bounded real valued functions on the positive integers $N$. If $f \in C^{*}(N), f^{\prime}$ is its extension to $\beta N$, the Stone-Cech compactification of $N$, and $f^{*}$ the restriction of $f^{\prime}$ to the compact space $\beta N \backslash N$. If $V \subset N, V^{\prime}$ is its closure in $\beta N$, and $V^{*}=V^{\prime} \cap$ $(\beta N \backslash N) . V^{*} \neq \varnothing$ iff $V$ is infinite, and sets of the form $V^{*}(V \subset N)$ are a basis of clopen (=open and closed) sets for the topology of $N^{*}=\beta N \backslash N$. (See [R] for details.)

If $C\left(N^{*}\right)$ is the space of continuous real valued functions on $N^{*}$, then it is isomorphic to the quotient space $C^{*}(N) / c_{0}$, where $c_{0}$ is the space of real functions on $N$ with limit 0 . If $A=\left(a_{m n}\right)$ is a regular matrix operator on $C^{*}(N)$, then $A\left(c_{0}\right) \subset c_{0}$, and hence $A$ induces an operator $A^{*}$ on $C\left(N^{*}\right)$ by the formula $A^{*}\left(f^{*}\right)=(A f)^{*}\left(f \in C^{*}(N)\right)$. Note that $f^{*}=0$ iff $f \in c_{0}$, in which case $A f \in c_{0}$ as well. Hence $f^{*}=0$ implies $A^{*}\left(f^{*}\right)=(A f)^{*}=0$, and so $A^{*}$ is a well-defined linear operator on $C\left(N^{*}\right)$. From regularity of $A$ it follows easily that $\left(A^{*} e\right)(x)=1$ for all $x \in N^{*}$, where $e(x)=1$ for all $x \in N^{*}$. If $C_{A}$ is the bounded convergence field of $A$, let $\left(C_{A}\right)^{*}=\left\{f^{*}: f \in C_{A}\right\}$. Then $\left(C_{A}\right)^{*}=\left\{f \in C\left(N^{*}\right): A^{*} f=\right.$ constant $\}$.

2.2. Definition. $M_{A}$ is the set of $g \in C^{*}(N)$ such that $\lim (n \rightarrow \infty)$ $\{A(g f)(n)-(A g)(n)(A f)(n)\}=0$ for all $f \in C^{*}(N) .\left(M_{A}\right)^{*}=\left\{g^{*}: g \in M_{A}\right\}$.

In [A] it was shown that $M_{A}$ and $\left(M_{A}\right)^{*}$ are Banach algebras, and that $g \in\left(M_{A}\right)^{*}$ iff $A^{*}(g f)=\left(A^{*} g\right)\left(A^{*} f\right)$ for all $f \in C\left(N^{*}\right)$.

2.3. Definition. A linear subspace $L$ of $C\left(N^{*}\right)$ is called large if for each nonvoid clopen $V \subset N^{*}$, there exists $f \in L$ such that $f(x)=0$ and $f(y)=1$ for some $x \in V$ and $y \in V$.

2.4. LEMMA. Let $A^{r}(r \in N)$ be regular matrices, $M^{r}$ the corresponding algebras as in 2.2, and $M=\bigcap\left\{M^{r}: r \in N\right\}$. Then $M^{*}$ is large, and so is $\left(A^{r}\right)^{*}\left(M^{*}\right)$ for each $r$ (where $\left.\left(A^{r}\right)^{*}\left(M^{*}\right)=\left\{\left(A^{r}\right)^{*}(f): f \in M^{*}\right\}\right)$.

Proof. First we consider the case of a single matrix $A=\left(a_{m n}\right)$, which we assume without loss of generality to be in truncated form, i.e., there exist $m(1)<m(2)<\cdots$ and $n(1)<n(2)<\cdots$ such that $m \in[m(k), m(k+1))$ implies $a_{m n}=0$ whenever $n \notin[n(k), n(k+2))$. (See [ $\mathbf{P}_{2}$, p. 82] or [E-P].) We may also assume each row sum is 1 . Let $t(n)$ be any bounded sequence of reals such that $\varepsilon(n)=t(n+1)-t(n) \rightarrow 0$, and define $g \in C^{*}(N)$ by $g(r)=$ $t(k)$ whenever $r \in[n(k), n(k+1))$. We show that $g \in M_{A}$. If $f \in C^{*}(N)$ and $m \in[m(k), m(k+1))$,

$$
\begin{aligned}
\sum_{j} a_{m j} g(j) f(j) & =t(k) \sum_{j=n(k)}^{n(k+1)-1} a_{m j} f(j)+t(k+1) \sum_{j=n(k+1)}^{n(k+2)-1} a_{m j} f(j) \\
\cdot \quad & =t(k) \sum_{j=n(k)}^{n(k+2)-1} a_{m j} f(j)+\varepsilon(k) \sum_{j=n(k+1)}^{n(k+2)-1} a_{m j} f(j) .
\end{aligned}
$$


Letting $f=1$ and recalling that row sums are 1 , we have

$$
t(k)=\sum_{j} a_{m j} g(j)-\varepsilon(k) \sum_{j=n(k+1)}^{n(k+2)-1} a_{m j} .
$$

Putting this value of $t(k)$ into (*) gives

$$
\begin{aligned}
\sum_{j} a_{m j} g(j) f(j)=\left[\sum_{j} a_{m j} g(j)-\varepsilon(k) \sum_{j=n(k+1)}^{n(k+2)-1} a_{m j}\right] \\
\cdot\left[\sum_{j=n(k)}^{n(k+2)-1} a_{m j} f(j)\right]+\varepsilon(k) \sum_{j=n(k+1)}^{n(k+2)-1} a_{m j} f(j) .
\end{aligned}
$$

It follows that

$$
\sum_{j} a_{m j} g(j) f(j)-\left(\sum_{j} a_{m j} g(j)\right)\left(\sum_{j} a_{m j} f(j)\right) \rightarrow 0 .
$$

Hence $g \in M_{A}$.

Let $V \subset N$ be infinite. We shall find $g \in M_{A}$ such that $g^{*}$ takes the values 0 and 1 on the clopen set $V^{*}$. But $V$ must meet an infinite number of the intervals [ $n(k), n(k+1)$ ), so by choosing $t(k)$ to be 0 and 1 infinitely often for $k$ such that $V$ meets $[n(k), n(k+1))$, and also so that $t(n+1)-t(n) \rightarrow 0$, we obtain (as above) a $g$ as desired (cf. $\left[\mathbf{P}_{1}\right]$ ). Clearly, the extension $g^{*}$ takes the values 0 and 1 on $V^{*}$. A similar construction will give $g$ such that the transform $\mathrm{Ag}$ does the same.

The above construction of the function $g$ depended only on the particular way the matrix $A$ was truncated. Hence to complete the proof it suffices to show that the matrices $A^{r}(r \in N)$ can be 'simultaneously truncated' (cf. $\left[\mathbf{P}_{2}\right.$, p. 95]). We give the first few steps of an algorithm for achieving this. Let $m(1)=n(1)=1$, and let $n(2)>n(1)$. By regularity of $A^{1}$ and $A^{2}$ we choose $m(2)>m(1)$ such that $m \geqq m(2)$ implies $\sum\left\{\left|a_{m n}^{r}\right|: n \leqq n(2)\right\}<\frac{1}{2}$ $(r=1,2)$. Now choose $n(3)>n(2)$ such that $m(1) \leqq m<m(2)$ implies $\sum\left\{\left|a_{m n}^{r}\right|: n(3) \leqq n\right\}<\frac{1}{2}(r \in 1,2)$. Choose $m(3)>m(2)$ such that $m \geqq m(3)$ implies $\sum\left\{\left|a_{m n}^{r}\right|: n \leqq n(3)\right\}<\frac{1}{3}(r=1,2,3)$. Choose $n(4)>n(3)$ such that $m(2) \leqq m<m(3)$ implies $\sum\left\{\left|a_{m n}^{r}\right|: n(4) \leqq n\right\}<\frac{1}{3} \quad(r=1,2,3)$. And so on. (Cf. the picture in [E-P].) Every matrix $A^{r}$ will have truncated form beginning with some row, and it is easy to see that rows may be adjusted to have sum 1 without affecting limiting behavior at infinity.

3. The consistency theorem. If $A$ is an infinite matrix such that $A\left(c_{0}\right) \subset c_{0}$, then it is easy to see that regularity of $A$ is equivalent with $A^{*} e=e$, where $e$ is the unit function in $C\left(N^{*}\right)$. We have also noted that if $C_{A}$ is the bounded convergence field of $A$, then $\left(C_{A}\right)^{*}=\left\{f \in C\left(N^{*}\right): A^{*} f=\right.$ const $\}$, and $C_{A} \subset C_{B 3}$ iff $\left(C_{A}\right)^{*} \subset\left(C_{B}\right)^{*}$. Thus facts about matrix operators 
on $C^{*}(N)$ translate into facts about the induced operators on $C\left(N^{*}\right)$ and vice versa. For the remainder of the paper our results are stated in terms of the induced operators on $C\left(N^{*}\right)$, leaving the relevant translations to the reader.

3.1. Topological Facts. (a) A nonvoid $G_{\delta}$ set in $N^{*}$ always has nonvoid interior. (b) A nonvoid open set in $N^{*}$ cannot be expressed as a union of a collection of cardinality $\boldsymbol{\aleph}_{1}$ of closed nowhere dense sets.

For fact (a), see [R]. (b) follows from (a) [PI, p. 46]; and, as Plank notes, this fact is implicit in [R].

3.2. Definition. Let $L$ be a linear subspace of $C\left(N^{*}\right)$. Two points $x$, $y \in N^{*}$ are equivalent under $L$ if $f(x)=f(y)$ for all $f \in L . L$ is called small if there exist at most $\boldsymbol{\aleph}_{1}$ equivalence classes under $L$.

Since $N^{*}$ has $2^{c}$ points [R], $C\left(N^{*}\right)$ is not small. Obviously, the space of constant functions is small. A less trivial example: let $f \in C\left(N^{*}\right)$ have finite or countable range, and let $L$ be the Banach subalgebra of $C\left(N^{*}\right)$ generated by $f$.

If we assume the continuum hypothesis $c=\aleph_{1}$, then the Banach subalgebra of $C\left(N^{*}\right)$ generated by any finite or countable collection of elements is small.

3.3. Definition. If $L$ is a linear subspace of $C\left(N^{*}\right)$ and $A$ a regular matrix, $C_{A L}=\left\{f \in C\left(N^{*}\right): A^{*} f \in L\right\}$. Note that if $L=$ constant functions, then $C_{A L}=\left(C_{A}\right)^{*}$, where $C_{A}$ is the bounded convergence field of $A$ and $\left(C_{A}\right)^{*}=\left\{f^{*}: f \in C_{A}\right\}$.

3.4. Consistency Theorem. Let $A$ and $B$ be regular matrices and $L a$ small linear subspace of $C\left(N^{*}\right)$. Suppose that for each $k \in L$, there exists $h \in L$ such that $A^{*} h=B^{*} h=k$. Then $C_{A L} \subset C_{B L}$ implies $A^{*} f=B^{*} f$ for all $f \in C_{A L}$.

Proof. Let $f_{1} \in C_{A L}, A^{*} f_{1}=k$, and suppose $B^{*} f_{1}=p \neq k$. We show that this implies $C_{A L} \not \subset C_{B L}$. Choose $h \in L$ such that $A^{*} h=B^{*} h=k$, and let $f=f_{1}-h$. Then $A^{*} f=0 \in L$, and $B^{*} f=p-k=j \in L \backslash\{0\}$. Choose real $s \neq 0$ such that $j^{-1}(s)$ is nonvoid. Then $j^{-1}(s)$ is a closed $G_{\delta}$ set, so 3.1 (a) implies $j^{-1}(s)$ contains a nonvoid clopen set $W$. Now $W$ meets at most $\boldsymbol{\aleph}_{1}$ equivalence classes under $L$, so by 3.1 (b) at least one such class contains a clopen set $V$. By Lemma 2.4 there exists $g \in\left(M_{A}\right)^{*} \cap\left(M_{B}\right)^{*}$ such that $B^{*} g$ takes the values 0 and 1 on $V$. Then $A^{*}(f g)=\left(A^{*} f\right)\left(A^{*} g\right)=0 \in L$, while $B^{*}(f g)=\left(B^{*} f\right)\left(B^{*} g\right)=s\left(B^{*} g\right)$ on $V$. Since $B^{*} g$ is not constant on $V$ and $V$ is contained in a set of constancy for $L$, it follows that $B^{*}(f g) \notin L$. Hence $f g \in C_{A L} \backslash C_{B L}$, and $C_{A L} \not C_{B L}$.

3.5. REMARK. A simple situation where the hypotheses of 3.4 hold is when $A^{*}(L)=B^{*}(L)=L$, and $A^{*} f=B^{*} f=f$ for all $f \in L$. By regularity of $A$ and $B$, this includes the case where $L=$ constant functions. 
4. Applications to multiplicative summability theory. For the classical case $L=$ constant functions, Theorem 4.2 was proved by Hill and Sledd [H-S, p. 412]. The main idea in the proof of 4.1 occurs in their paper as well.

4.1. TheOrem. Let $L$ be a small subalgebra of $\left(M_{A}\right)^{*}$ such that (a) $e \in L$, (b) if $f \in L$ and $f$ has no zeros, then $f^{-1} \in L$, and (c) $L \subset\left(A^{*}\right)(L)$. If $g C_{A L} \subset C_{A L}$, then $A^{*}(f g)=\left(A^{*} f\right)\left(A^{*} g\right)$ for all $f \in C_{A L}$.

Proof. Since $e \in C_{A L}$, we have $g \in C_{A L}$, so $A^{*} g \in L$. Assume

Case I. $A^{*} g=k$ has no zeros. Then $k^{-1} \in L$, and by (c) there is $h \in L$ with $A^{*} h=k^{-1}$. Let the operator $B^{*}$ be defined on $C\left(N^{*}\right)$ by $B^{*} f=$ $A^{*}(h g f)=\left(A^{*} h\right) A^{*}(g f)=k^{-1} A^{*}(g f)$, the next to the last equality because $h \in L \subset\left(M_{A}\right)^{*}$. The operator $B^{*}$ on $C\left(N^{*}\right)$ is induced by the matrix operator $B$ on $C^{*}(N)$ defined as follows: let $g_{1}$ and $h_{1}$ in $C^{*}(N)$ be such that $g=\left(g_{1}\right)^{*}$ and $h=\left(h_{1}\right)^{*}$. Then $B f=A\left(h_{1} g_{1} f\right)\left(f \in C^{*}(N)\right)$. $B$ is regular, since $B^{*} e=k^{-1} A^{*} g=k^{-1} k=e$.

We show that the pair $A, B$ satisfies the conditions of Theorem 3.4. First, $C_{A L} \subset C_{B L}$, because $f \in C_{A L}$ implies $f g \in C_{A L}$ implies $B^{*} f=$ $A^{*} h A^{*}(f g) \in L L \subset L$ ( $L$ is an algebra) implies $f \in C_{B L}$. Secondly, if $u \in L$, then by (c) there is $v \in L \subset\left(M_{A}\right){ }^{*}$ with $A^{*} v=u$, and then $B^{*} v=k^{-1} A^{*}(g v)=$ $k^{-1}\left(A^{*} g\right)\left(A^{*} v\right)=k^{-1} k u=u$. Thus $A^{*} v=B^{*} v=u$.

It now follows from Theorem 3.4 that for all $f \in C_{A L}, A^{*} f=B^{*} f=$ $k^{-1} A^{*}(g f)$, or $A^{*}(g f)=\left(A^{*} g\right)\left(A^{*} f\right)$.

Case II. If $A^{*} g=k$ and $k$ has zeros, let $\rho$ be a constant such that $A^{*}(g+\rho e)=k+\rho e$ has no zeros, and let $g^{\prime}=g+\rho e$. Then $g^{\prime} C_{A L} \subset C_{A L}$, so by what we have already shown, $A^{*}\left(g^{\prime} f\right)=\left(A^{*} g^{\prime}\right)\left(A^{*} f\right)$ for all $f \in C_{A L}$. Hence

$$
\begin{aligned}
A^{*}(g f)+\rho\left(A^{*} f\right) & =A^{*}(g f+\rho f)=A^{*}\left(g^{\prime} f\right)=\left(A^{*} g^{\prime}\right)\left(A^{*} f\right) \\
& =\left(A^{*} g+\rho\right) A^{*} f=\left(A^{*} g\right)\left(A^{*} f\right)+\rho\left(A^{*} f\right),
\end{aligned}
$$

or $A^{*}(g f)=\left(A^{*} g\right)\left(A^{*} f\right)$.

4.2. THEOREM. Let $L$ be as in 4.1 , and assume $A^{*} \geqq 0$. The following are equivalent:

(a) $A^{*}(f g)=\left(A^{*} f\right)\left(A^{*} g\right)$ for all $f$ and $g$ in $C_{A L}$;

(b) $C_{A L} \subset\left(M_{A}\right)^{*}$;

(c) $C_{A L}$ is an algebra.

Proof. (a) implies (b). If $f \in C_{A L}$, then (a) implies $A^{*}\left(f^{2}\right)=\left(A^{*} f\right)^{2}$. Since $A^{*} \geqq 0,\left[\mathbf{A}\right.$, Theorem 1.3] implies $f \in\left(M_{A}\right)^{*}$.

(b) implies (c). Let $f$ and $g \in C_{A L}$. If (b) holds, then $A^{*}(f g)=$ $\left(A^{*} f\right)\left(A^{*} g\right) \in L L \subset L$, since $L$ is an algebra. Hence $f g \in C_{A L}$, so $C_{A L}$ is an algebra.

(c) implies (a). This follows from 4.1 . 
4.3. Remarks. (a) Positivity of $A^{*}$ was used only for the implication '(a) implies (b)'. (b) In the case $L=$ constant functions, it was shown in [A, Theorem 1.5] that $C_{A L} \cap\left(M_{A}\right) *$ is the set of functions constant on the socalled 'support set' of $A^{*}$, and so if $C_{A L} \subset\left(M_{A}\right)^{*}$, then the convergence field of $A$ has a particularly simple form.

4.4. THEOREM. Under the hypotheses of 4.1 , if $g C_{A L} \subset C_{A L}$ and $A^{*} g=0$, then $A^{*}\left(g^{+}\right)=A^{*}\left(g^{-}\right)=A^{*}(|g|)=0$ (where $g^{+}=\max (g, 0), g^{-}=\max (-g, 0)$, and $\left.|g|=g^{+}+g^{-}\right)$.

Proof. By regularity of $A, e \in C_{A L}$, hence $g \in C_{A L}, g^{2} \in C_{A L}$, and by induction $g^{k} \in C_{A L}$ for all $k$. Theorem 4.1 implies $A^{*}\left(g^{k}\right)=0$ for all $k$. Now as in the proof of the Stone-Weierstrass theorem $\left(N^{*}\right.$ is compact), $|g|$ is the uniform limit of polynomials $a_{1} g+a_{2} g^{2}+\cdots+a_{k} g^{k}$, so by continuity of $A^{*}$ we have $A^{*}(|g|)=0$. But $2 g^{+}=|g|+g$, and $2 g^{-}=|g|-g$, so $0=A^{*}\left(g^{+}\right)=A^{*}\left(g^{-}\right)$.

4.5. Remark. In [A, Theorem 1.5] it was shown that if $A^{*} \geqq 0, A^{*}$ is multiplicative on $\left(C_{A}\right)^{*}$ iff $\left(C_{A}\right)^{*}$ consists exactly of elements of $C\left(N^{*}\right)$ which are constant on the so-called support set $K$ of $A$. Theorem 4.4 leads to an interesting generalization of this. First, some notation: if $p \in N^{*}$, let $m_{p}$ be the regular Borel measure representing the functional $f \rightarrow$ $\left(A^{*} f\right)(p)\left(f \in C\left(N^{*}\right)\right), K_{p}$ the support set of $m_{p}$, and $K=$ closure $\bigcup\left\{K_{p}: p \in N^{*}\right\}$.

4.6. TheOREM. Let $A^{*} \geqq 0$, and let $L$ be a small subalgebra of $\left(M_{A}\right)^{*}$ such that (a) $e \in L$, (b) if $f \in L$ and $f$ has no zeros, then $f^{-1} \in L$, (c) $A^{*} f=f$ for each $f \in L$. The following are equivalent:

(1) $C_{A L}$ is an algebra;

(2) $C_{A L}$ consists exactly of the elements of $C\left(N^{*}\right)$ which agree on $K$ with some element of $L$.

When (1) and (2) hold, then $A^{*} f|K=f| K$ for each $f \in C_{A L}$.

Proof. (2) implies (1). Since $L$ is an algebra, it is obvious that the set of elements of $C\left(N^{*}\right)$ which agree on $K$ with some element of $L$ is an algebra.

(1) implies (2). First, let $f \in C_{A L}$ and $A^{*} f=k \in L$. Hypothesis (c) implies $A^{*}(f-k)=0$. Since $L$ is an algebra, 4.4 implies $0=A^{*}|f-k|$. Hence for each $p \in N^{*}, 0=\int|f-k| d m_{p}$. Since $m_{p}$ is a positive measure, $f=k$ on $K_{p}$, and hence on $K$. On the other hand, if $f$ agrees with $k \in L$ on $K$, then for each $p \in N^{*}, A^{*} f(p)=\int f d m_{p}=\int k d m_{p}=A^{*} k(p)=k(p)$, hence $A^{*} f=$ $k \in L$, so $f \in C_{A L}$.

The final assertion is now obvious. 


\section{REFERENCES}

[A] R. Atalla, On the multiplicative behavior of regular matrices, Proc. Amer. Math. Soc. 26 (1970), 437-446. MR 42 \#6633.

[A-B] R. Atalla and J. Bustoz, On sequential cores and a theorem of R. R. Phelps, Proc. Amer. Math. Soc. 21 (1969), 36-42. MR 39 \#4679.

[B] A. Brudno, Summation of bounded sequences by matrices, Mat. Sb. 16 (58) (1945), 191-247. (Russian) MR 7, 12.

[E-P] P. Erdös and G. Piranian, The topologization of a sequence space by Toeplitz matrices, Michigan Math. J. 5 (1958), 139-148. MR 21 \#812.

[H-S] J. D. Hill and W. T. Sledd, Approximation in bounded summability fields, Canad. J. Math. 20 (1968), 410-415. MR 36 \#5561.

[M-O $\mathrm{O}_{1}$ S. Mazur and W. Orlicz, Sur les méthodes linéaires de sommation, C. R. Acad. Sci. Paris 196 (1933), 32-34.

$\left[\mathrm{M}-\mathrm{O}_{2}\right]-$, On linear methods of summability, Studia Math. 14 (1954), 129-160. MR 16, 814.

$\left[\mathbf{P}_{1}\right]$ G. M. Petersen, Summability methods and bounded sequences, J. London Math. Soc. 31 (1956), 324-326. MR 18, 31.

$\left[\mathbf{P}_{\mathbf{2}}\right] \longrightarrow$, Regular matrix transformations, McGraw-Hill, New York, 1966. MR 37 \#642.

[PI] D. Plank, On a class of subalgebras of $C(X)$ with applications to $\beta X \backslash X$, Fund. Math. 64 (1969), 41-54. MR 39 \#6266.

[R] W. Rudin, Homogeneity problems in the theory of Cech compactifications, Duke Math. J. 23 (1956), 409-419. MR 18, 324.

Department of Mathematics, Ohio University, Athens, Ohio 45701 